\title{
Electronic Nose for Microbiological Quality Control of Food Products
}

\author{
M. Falasconi, ${ }^{1}$ I. Concina, ${ }^{1}$ E. Gobbi, ${ }^{1,2}$ V. Sberveglieri, ${ }^{3}$ A. Pulvirenti, ${ }^{3}$ and G. Sberveglieri ${ }^{1}$ \\ ${ }^{1}$ CNR-IDASC SENSOR Laboratory and University of Brescia, Via Valotti 9, 25133 Brescia, Italy \\ ${ }^{2}$ Department of Agricultural and Environmental Sciences, University of Udine, Via delle Scienze 208, 33100 Udine, Italy \\ ${ }^{3}$ Department of Agricultural and Food Sciences, Modena and Reggio Emilia University, Via Amendola 2, 42100 Reggio Emilia, Italy \\ Correspondence should be addressed to M. Falasconi, matteo.falasconi@ing.unibs.it
}

Received 7 September 2011; Revised 1 December 2011; Accepted 1 December 2011

Academic Editor: Miloslav Pravda

Copyright (c) 2012 M. Falasconi et al. This is an open access article distributed under the Creative Commons Attribution License, which permits unrestricted use, distribution, and reproduction in any medium, provided the original work is properly cited.

Electronic noses (ENs) have recently emerged as valuable candidates in various areas of food quality control and traceability, including microbial contamination diagnosis. In this paper, the EN technology for microbiological screening of food products is reviewed. Four paradigmatic and diverse case studies are presented: (a) Alicyclobacillus spp. spoilage of fruit juices, (b) early detection of microbial contamination in processed tomatoes, (c) screening of fungal and fumonisin contamination of maize grains, and (d) fungal contamination on green coffee beans. Despite many successful results, the high intrinsic variability of food samples together with persisting limits of the sensor technology still impairs ENs trustful applications at the industrial scale. Both advantages and drawbacks of sensor technology in food quality control are discussed. Finally, recent trends and future directions are illustrated.

\section{Introduction}

Aroma is one of the most significant parameters among the sensory properties of foods. The characteristic flavor of volatile compounds, so-called fingerprint, may provide information about safety and specific characteristics of food, acting sometimes as an indicator of process fault as well. Indeed, some volatile compounds can originate from biochemical processes of the food, as a consequence of technological treatments or product storage. Unwanted smells (off-flavours) may include substances originating from the metabolism of spoilage microorganisms, bacteria and fungi, which may naturally or accidentally contaminate the products prior or during its production [1].

Microbial contamination affects most of foodstuffs consumed in the world, often as a mandatory step of the food production chain. For instance, a residual bacterial charge is commonly accepted and even wished in some foods (for instance fermented milk and derivatives), and the fungal presence is a characteristic pursued in some cases (as for some cheese varieties and salami). Yet, in many cases the presence of unwanted microbial contaminants can be a serious problem depending on the nature and level of contamination.

Food containing pathogenic microorganisms can be extremely harmful for customer's health; while most foodborne diseases are sporadic and often not reported, foodborne disease outbreaks may take on massive proportions. For example, in 1994, an outbreak of salmonellosis due to contaminated ice cream occurred in the USA, affecting an estimated 224,000 persons. In 1988, an outbreak of hepatitis $\mathrm{A}$, resulting from the consumption of contaminated clams, affected some 300,000 individuals in China (WHO, Food safety and foodborne illness, Fact Sheet no. 237, 2007). Foodborne diseases are a widespread and growing public health problem, both in developed and developing countries. In industrialized countries, the percentage of the population suffering from foodborne diseases each year has been reported (WHO) to be up to $30 \%$. In the United States of America (USA), for example, around 76 million cases of foodborne diseases, resulting in 325,000 hospitalizations and 5,000 deaths, are estimated to occur each year, while in Europe every year $15 \%$ of the population become ill by contaminated food as reported by the European Food 
Safety Authority (EFSA). Food contamination creates an enormous social and economic burden on communities and their health systems. In the USA, diseases caused by the major pathogens alone are estimated to cost up to US \$35 billion annually in medical costs and lost productivity. The re-emergence of cholera in Peru in 1991 resulted in the loss of US $\$ 500$ million in fish and fishery product exports that year.

Apart from health problem, microorganisms can cause unacceptable organoleptic alterations of taste and flavor of the final products, resulting in economic damages for the food producers. The availability of reliable, fast, easyto-use tools for early screening of food microbiological contamination is therefore a target both for customer's safeguard and production improvement. Traditional quality control tests include microbiological and physical/chemical techniques (microbiological cell counting, gas and liquid chromatography, mass spectrometry, optical spectroscopic techniques). Although effective and accurate, these have some usual drawbacks, such as high costs of implementation, long time of analysis, low samples throughput, need of a highly qualified manpower, and cannot be used for online production monitoring. On the other side, trained human sensory panels, often employed for food quality assessment, are also not suitable for routine industrial controls because they suffer from lack of objectivity and reliability due to human fatigue or stress, requiring long training time and high implementation costs.

Chemical- and biosensor technologies have recently emerged as valuable candidates for food quality control $[2,3]$ due to their simplicity of use, low cost, rapidity, and good correlation with sensory panels. Electronic noses (ENs) are instruments based on an array of semiselective gas sensors and pattern-recognition methods $[4,5]$.

ENs have been applied in various food contexts, such as process monitoring, freshness evaluation, shelf-life investigation, authenticity determination, and product traceability. Those applications have been extensively reviewed in the literature [6-12]; however, little emphasis has been given until now to microbiology applications of chemical sensor devices, for instance, the screening of foodborne pathogens contamination.

Many recent works have evidenced that ENs can be exploited to screen microbial contamination of food by analyzing the pattern of volatile compounds produced by microbial metabolism. The fingerprint variation can be due to either the appearance of new chemical compounds (primary or secondary metabolites) or to changes in the relative amount of the original volatile compounds without changes in the qualitative composition. Detection of foodcontamination by using standard microbial plate count methods involves time and extensive sample preparation. Also, improper sampling of the food product may give misleading results since the culture-based methods rely on the site of sampling. The use of EN can provide rapid and accurate means of sensing the incidence of food contaminant bacteria with little or no sample preparation.

Therefore, this review paper aims to present an overview of recent results achieved by applying ENs in the field of food microbiology. Emphasis will be given to four paradigmatic case studies in which a metal oxide sensors EN has been used, namely (a) the screening of Alicyclobacillus spp. spoilage of fruit juices (b) the early detection of microbial contamination in processed tomatoes (c) the screening of fungal and Fumonisin contamination of maize grains and (d) the fungal contaminations of green coffee beans.

\section{Microbiology Applications of ENs}

In the food safety framework, the use of EN devices for rapid and reliable testing of pathogenic bacteria contamination in foods is widespread. ENs have been successfully used to detect spoilage of a large variety of food categories and food products as shown in Table 1. The table reports the application of various chemical sensor systems, based on different sensor technologies, catalogued by food category (down to food products) and nature of the screened contaminants (either microbial or toxins).

Several applications are reported for quality control of grains by odour mapping techniques. These controls are routinely performed by a human olfactory panel constituted by inspectors smelling the grain odour. EN could then represent a valid method of choice for its rapidity, simplicity, and low cost. More importantly, this would overcome the potential health hazard to the human tasters caused by repeated exposures to moulds spores and mycotoxins and the low predictive ability of odour classification system for certain mycotoxins contamination. Hybrid sensor technology has shown to be able determining the mycological quality of barley grains [16] and wheat $[17,18]$, as well as to detect some mycotoxins classes such as Fumonisin and Aflatoxin $[17,19]$. Much emphasis has been given to maize grains, whose contamination by mycotoxigenic fungi such as fusaria and by fumonisins, the mycotoxins they produce, has been thoroughly investigated $[15,20]$. More on this subject will be presented in the case study (c).

Similar to cereal grain contamination is that related with coffee grains or beans. EN has been widely applied in the past along the entire coffee production chain. For instance, various EN sensing technologies have been used to distinguish different types of coffee beans, to identify various brands and mixtures, or again to classify commercial coffee blends and samples with different roasting levels ([21] and references therein). Ever, the investigation of microbial contamination of coffee grains has received little attention. This application is referred in the case study (d).

Fungal spoilage is an important issue in bakery products. Some companies use the measurement of the water activity of the final products as an index for fungal spoilage prediction and batches rejection. However, the need for reliable alternative methods has been evidenced. Marín et al. [22] used an MS-based electronic nose to detect fungal spoilage in samples of bakery products. These were inoculated with different Eurotium, Aspergillus, and Penicillium species. Once the headspace was sampled, ergosterol content was determined in each sample in order to have a reference technique for EN training and testing. Both the EN signals and ergosterol levels were used to build models for prediction 
TABLE 1: Applications of chemical sensors in the food microbiology sector.

\begin{tabular}{|c|c|c|c|c|}
\hline Food category & Specific product & Instrumental system and/or sensor technology & $\begin{array}{l}\text { Nature of the } \\
\text { contamination }\end{array}$ & Reference \\
\hline \multirow{8}{*}{ Grains } & \multirow[t]{2}{*}{ Maize } & $\begin{array}{l}\text { EOS835, Sacmi } \\
\text { (thin film MOX) }\end{array}$ & Fungi and fumonisins & {$[15,20]$} \\
\hline & & MOX & Aflatoxins & [19] \\
\hline & \multirow[t]{2}{*}{ Maize } & $\begin{array}{c}\text { LibraNose } \\
\text { (quartz Microbalances) }\end{array}$ & Fungi & [17] \\
\hline & & Polymers & Fungi & [18] \\
\hline & \multirow{3}{*}{ Barley } & Cyranose-320 (carbon-black polymer sensors) & Fungi & [38] \\
\hline & & $\begin{array}{l}\text { VCM } 422 \text { S-SENCE MOSFET, tin oxide } \\
\text { Taguchi sensors }\end{array}$ & Fungi & [16] \\
\hline & & $\begin{array}{l}\text { VCM } 422 \text { S-SENCE MOSFET, tin oxide } \\
\text { Taguchi sensors }\end{array}$ & Fungi, Ochratoxin A & [39] \\
\hline & Oats rye and barley & MOSFET and Taguchi & Fungi and bacteria & {$[40]$} \\
\hline \multirow{3}{*}{ Bakery products } & Bakery analogous & MS e-nose & Fungi & {$[22]$} \\
\hline & Bread analogous & Bloodhound BH-114 & $\begin{array}{l}\text { Bacteria, yeasts, and } \\
\text { fungi }\end{array}$ & [23] \\
\hline & Beef strip loins & Cyranose-320 (Carbon-black polymer sensors) & Bacteria & {$[24]$} \\
\hline \multirow[t]{4}{*}{ Meat } & Beef strip loins & Taguchi sensors & $\begin{array}{l}\text { Bacteria (Salmonella } \\
\text { typhimurium) }\end{array}$ & {$[25]$} \\
\hline & Beef and sheep meat & Taguchi sensors & Bacteria & [26] \\
\hline & Pork & MOX & Bacteria & [27] \\
\hline & Sardines & Doped tin oxide & Bacteria & [31] \\
\hline \multirow[t]{3}{*}{ Fish } & $\begin{array}{l}\text { Alaska pink salmon } \\
\text { (Oncorhynchus gorbuscha) }\end{array}$ & $\begin{array}{c}\text { Cyranose } 320 \\
\text { (carbon-black polymer sensors) }\end{array}$ & Bacteria & {$[30]$} \\
\hline & $\begin{array}{l}\text { Fresh Atlantic salmon } \\
\quad \text { (Salmo salar) }\end{array}$ & $\begin{array}{c}\text { AromaScan } \\
\text { (conductive polymers) }\end{array}$ & Bacteria & {$[29]$} \\
\hline & $\begin{array}{l}\text { Cold-smoked Atlantic } \\
\text { salmon (Salmo salar) }\end{array}$ & FishNose (GEMINI-6 MOS sensor) & Bacteria & {$[28]$} \\
\hline \multirow{4}{*}{ Milk and dairy product } & Milk & MOX, MOSFET & Bacteria & [41] \\
\hline & Ewe milk & MOX, MOSFET & Aflatoxin B1 & {$[32]$} \\
\hline & Milk & Polymer sensors & Bacteria, yeasts & {$[42]$} \\
\hline & Milk & MOX & Bacteria & {$[14]$} \\
\hline \multirow{3}{*}{$\begin{array}{l}\text { Processed } \\
\text { vegetables/fruit }\end{array}$} & Onion & $\begin{array}{l}\text { Smith Detection Inc., Pasadena, CA } \\
\text { (Polymer sensors) }\end{array}$ & Bacteria, fungi & [33] \\
\hline & Tomatoes & EOS835, Sacmi (thin-film MOX) & Fungi, bacteria, yeasts & {$[34]$} \\
\hline & Fruit juices & EOS835, Sacmi (thin-film MOX) & $\begin{array}{c}\text { Bacteria } \\
\text { (Alicyclobacillus) }\end{array}$ & {$[13,35]$} \\
\hline \multirow[t]{2}{*}{ Drinks } & Soft drinks & EOS835, Sacmi (thin-film MOX) & $\begin{array}{c}\text { Bacteria } \\
\text { (Alicyclobacillus) }\end{array}$ & [36] \\
\hline & Red wine & FOX 3000 Alpha MOs (MOX sensors) & Yeast (Brettanomyces) & [37] \\
\hline
\end{tabular}

of ergosterol content. This model has shown excellent regression performance (between 87 and 96\% in some cases) confirming the EN as a reliable method.

Needham and coworkers [23] applied a commercial EN (Bloodhound BH-114) for early detection and differentiation of both bacteria (Bacillus subtilis) and fungi (Penicillium verrucosum and Pichia anomala) spoilage of bread analogues. Cluster analysis led in this case a differentiation between microbial and physiological (lipoxygenase) spoilage after $48 \mathrm{~h}$.

Much work has been done in the electronic detection of quality characteristics of meat products within the food industry. Meat is an ideal growth medium for several groups of pathogenic bacteria (such as Salmonella, Escherichia coli, or Listeria monocytogenes). Estimation of meat safety and quality is usually based on microbial cultures. Bacterial strain identification requires a number of different growth conditions and biochemical tests with overnight or large incubation periods and skilled personnel, which means that testing may not be frequently performed.

In [24], Panigrahi and co-workers, analysed the headspace from fresh beef strip loins kept at $4^{\circ} \mathrm{C}$ for 10 days by a commercially available Cyranose- 320 with conducting polymer sensors. They developed various classification models using radial basis function neural networks that enable to identify (with accuracies of $100 \%$ ) spoiled and unspoiled meat samples. In this case the type of bacteria was not identified, but results were correlated with total 
viable counts (TVCs). The same research group [25] also investigated the ability of home-made EN (Taguchi sensors based) for screening the contamination of beef samples by Salmonella typhimurium. In this case the results obtained suggested that the use of higher-order statistical techniques, like independent component analysis (ICA), could help in improving the performance of the sensor system. Other groups, like El Barbri et al. [26], analyzed both beef and sheep meat (stored at $4^{\circ} \mathrm{C}$ for up to 15 days) by a laboratory EN based on Taguchi sensors aiming to develop a protocol for the quality control of red meat. The EN, coupled to SVM, could discriminate between unspoiled/spoiled beef or sheep meats with a success rate above $96 \%$. Good correlation between the EN signals and the bacteriological data were also obtained. Wang et al. [27] used an EN equipped with MOX sensors together with support vector machine (SVM) and partial least squares (PLSs) to predict the total viable counts in chilled pork samples. The achieved correlation coefficients for training and validation were close to $90 \%$, which suggested that the EN system could be used as a simple and rapid technique for absolving the task.

Most freshness and spoilage investigations with ENs have involved studies with fish or fish products.

Quality changes of cold-smoked salmon from four different smokehouses in Europe were monitored by a prototype MOS sensors array system, the so-called FishNose [28]. The responses of the gassensors correlated well with sensory analysis of spoilage odor and microbial counts suggesting that they can detect volatile microbially produced compounds causing spoilage odors in cold-smoked salmon during storage. In this case, gassensor selection was optimized for the detecting of changes in the highly volatile compounds mainly representing microbial metabolism during spoilage. The system was therefore ideal for fast quality control related to freshness evaluation of smoked salmon products.

Regarding fresh salmon fillets, the feasibility of using an AromaScan EN to assess seafood quality and microbial safety was assessed by Du et al. [29]. AromaScan mappings of these fillets were compared to their time-related changes in microbial counts, histamine contents, and sensory panel evaluations. Promising results were obtained, and authors concluded that the EN can be used as an assisting instrument to a sensory panel in evaluating the seafood microbial quality and safety.

The ability of a portable hand-held EN (Cyranose $320 \mathrm{TM}$, composed of 32 individual thin-film carbon-black polymer sensors) in detecting spoilage of salmon under different storage conditions (at $14^{\circ} \mathrm{C}$ and in slush ice) was also investigated by Chantarachoti et al. [30]. As a result of these experiments, a predictive model may be developed for spoilage of whole Alaska pink salmon by analyzing belly cavity odors using the e-nose. This could be easily extended to other types of fish. In fact, an EN system based on a 4element, integrated, micromachined, MOX gas sensor array was used in [31] to assess the evolutionary stages of freshness in sardine samples stored up to one week at $4^{\circ} \mathrm{C}$.

Research with ENs in the area of milk and other dairy products has ranged from detecting adulteration/contamination of milk to determining the geographical origins of cheese. An important aspect related to milk quality and safety is the detection of contaminants, including aflatoxins, in milk. For instance, Benedetti et al. [32] studied the feasibility of using a commercial sensor array system, comprising 12 MOS and 12 MOSFET sensors, to detect the presence of aflatoxin M1 (AFM1). In this study, twenty-four raw milk samples, collected from two different groups of ewes fed with a formulated feed containing increasing amounts of aflatoxin B1, and six non-contaminated ewe milk samples were analysed. The results obtained by using the head space sensor array, processed by statistical methods, made it possible to group the samples according to the presence or the absence of aflatoxin M1.

Finally, various promising results have been also achieved with EN for microbial screening of fresh and processed vegetables, like onions [33] and tomatoes [34], fruit juices $[13,35]$ and drinks $[36,37]$.

\section{EOS Electronic Nose Description}

The electronic nose EOS (SACMI IMOLA scarl, Imola, Italy) [4] has been extensively used in the past in various application fields including the case studies that will be illustrated in the next sections. It consists of a pneumatic assembly for dynamic sampling (pump, electrovalve, electronic flow meter), a thermally controlled sensor chamber of $20 \mathrm{~mL}$ internal volume, an electronic board for controlling the sensor heaters and measuring the sensing layers, and software for data acquisition and signal processing. The instrument remote control and the data acquisition can be performed by an external laptop through standard communication port RS232. Two models are currently available: EOS835 and EOS507; the latter being a recent upgrade of the system with a humidity control device of the baseline air (based on a Peltier cell) and a more accurate sensor read-out electronics (up to $1 \mathrm{Gohm}, 10 \mathrm{~Hz}$ acquisition frequency).

EOS array is equipped with six metal oxide sensors. These can be both thick-film commercial Taguchi sensors (TGS2611 and TGS2442 were employed for instance by Cagnasso et al. [13]) or home-made thin-film sensors (further details can be found in Comini et al. [60] and references therein) among which tin oxide, often catalyzed with noble metals (e.g., Au, Ag, Pt, Pd, or Mo), and other metal oxides like tungsten oxide are commonly used.

The EOS supports dynamic or static headspace sampling unit (optionally with an autosampler HT200H, HTA srl, Italy). Static headspace has clear advantages in terms of reproducibility and repeatability. The HS generation parameters (incubation temperature, time, and so on) can be fully and accurately controlled. Besides, the HS analysis is carried out without perturbing the equilibrium conditions-this ensures there are no artefacts in the sensor response due to changes of HS concentration during the measurement. Finally, static headspace may be used to perform long runs of measurements, thus improving the training set collection and the device calibration. Nevertheless, the use of static headspace sampling strongly limits the EN sensitivity due to the small amount of available headspace (about $5 \mathrm{~mL}$ ) and consequently low carrier flow rate values $(10 \mathrm{~mL} / \mathrm{min})$. 
Therefore, in some applications, dynamic headspace is to be preferred; it basically consists of a pump and a flow controller that conveys the odour sample under investigation from a vessel (typically $100 \mathrm{~mL}$ in volume) into the sensor array chamber. As shown in previous works [13], sensitivity can be enhanced by one order of magnitude and sensor recovery time can be correspondently halved by using dynamic headspace.

Data generated with the EOS are elaborated by Exploratory Data Analysis (EDA) software, a written-in house software package based on MATLAB [37]. The EDA software includes the usual (univariate or multivariate) descriptive statistics functions among which principal component analysis (PCA) [44], with the additional utilities for easy data manipulation (e.g., data subsampling, dataset fusion) and plots customization.

Supervised classification is typically carried out by different pattern recognition algorithms. In the referred case studies, support vector machines (SVMs) with linear kernel [45] and k-Nearest Neighbour (1NN) classifier were applied. Fivefold cross-validation (CV) is usually implemented to get more robust classification results. Supervised regression is performed by partial least squares (PLSs) [46]; this technique can be used for training the EN to predict the colonyforming unit concentration (expressed as $\mathrm{cfu} / \mathrm{mL}$ ), taking as covariates the results of microbiological counts.

\section{Case Studies}

(a) Alicyclobacillus spp. Spoilage of Fruit Juices. In 1982, spoilage of aseptically filled apple juice from Germany was attributed to a new type of thermophilic acidophilic bacteria, later classified into a new genus, named Alicyclobacillus (ACB) [47]. Alicyclobacillus spp. are aerobic, Gram-positive, endospore-forming, nonpathogenic, thermoacidophilic bacteria isolated mainly from soil and hot springs. The spores are resistant to high temperature; thus they can survive ordinary pasteurization regimes used in the juice industry.

Contamination by Alicyclobacillus spp. was firstly detected in apple juice, but, since then, a larger variety of fruit juices, soft drink, fortified with minerals products have been found to be contaminated. A. acidoterrestris and A. acidocaldarius are the most common species able to cause typical off-flavours (medicine-like taints) in fruit juices; this was related to the production of 2-methoxyphenol (guaiacol), 2-6-dibromophenol, 2-6-dichlorophenol [48, 49] which are retained to be the markers of contamination.

Spoilage of fruit juices by Alicyclobacilli is extremely difficult to be revealed at early stages; therefore, this task is regarded as a relevant industrial issue that requires effective control measures to be developed. Culture-dependent conventional microbiological methods present some drawbacks, mainly related to the high detection limit and to the underestimation of the true microbial community. Traditionally, gas chromatography (GC) and mass spectrometry (MS) provide accurate measurements of the volatile fraction and are useful for specific identification of off-flavours compounds; nevertheless these methods remain still rather complex and expensive, being more suitable for laboratory quality control than for routine industrial analyses which often require faster, simpler, and massive screening of large product batches.

Different types of commercial fruit juices (orange, peach, pear, and apple) artificially contaminated by Alicyclobacillus spp. were tested with the EOS in an experimental work that lasted for almost two years.

Preliminary results reported in Gobbi et al. [35] were very promising, showing that EOS has good detection capabilities, being able to early reveal the presence of Alicyclobacillus spp. just after a growth time of 24 hours. The sensors showed some specificity related with the juice matrix; indeed contamination was easily identified in orange and peach juices (where detection threshold was around $100 \mathrm{cfu} / \mathrm{mL}$ ) whereas it was impossible to correctly classify the contaminated apple juice samples.

The juice samples were contaminated both with $A$. acidoterrestris and $A$. acidocaldarius to test whether intragenus specificity could affect the EOS results. In fact the peach juice samples contaminated by the two species were clustered closely together, but it was still possible perfectly to discriminate the contaminated samples from not contaminated ones. Thus, the intragenus specificity of EOS was much lower than the genus specificity. This can be an advantage because from a practical point of view it is possible to perform the EN training to diagnose bacterial contamination over a limited numbers of species without having the classification capability substantially affected by other species.

These results were subsequently confirmed and ameliorated by Cagnasso et al. [13]. The EOS showed good classification performance of contaminated samples (Table 2), up to $90 \%$ for pear juices classified by SVM. The system detection limits and the required growth times were consistent with the results for orange and apple juices formerly obtained by Gobbi et al. and still appeared to be specific of the juice matrix with apple juice being the most challenging case. Following these results, the authors argued that the detection of Alicyclobacillus spp. was favored by the strong change of specific volatile compounds present in the juice matrix, for instance, limonene on orange juice, although this hypothesis was not assesse through dedicated analytical studies.

Very fast though not very accurate capability to predict the amount of contamination was also observed (Figure 1). The number of cfu/mL predicted by the EOS correlated quite well with the true value as measured by the microbiological essays (the correlation coefficient scored about 0.80 ). The discrepancies can be associated with three facts: first, the EOS overestimated the $\mathrm{cfu} / \mathrm{mL}$ at low values, the predicted mean value are about 3 times larger; second, the PLS model has been built with the mean $\log (\mathrm{cfu} / \mathrm{mL})$ value whilst the EOS measurements refer to the actual concentration of individual samples which is not known; third, the model suffers from the accuracy with which the microbiological counts are determined that can be realistically of one order of magnitude.

EOS results were also correlated with GC-MS quantification of the claimed chemical markers associated with $A$. acidoterrestris presence, that is, guaiacol, but outcomes were inconsistent. The authors then argued that the gas sensors 
TABLE 2: Classification results of contaminated samples and detection thresholds (reproduced from [13]).

\begin{tabular}{llccc}
\hline $\begin{array}{l}\text { Type of } \\
\text { juice }\end{array}$ & SVM & $1 \mathrm{NN}$ & $\begin{array}{c}\text { Detection thresholds } \\
\text { Growth time } \\
\text { (hours) }\end{array}$ & $\mathrm{cfu} / \mathrm{mL}$ \\
\hline Orange & $86 \%$ & $78 \%$ & 24 & $10^{3}$ \\
Pear & $90 \%$ & $84 \%$ & 24 & $10^{2}-10^{3}$ \\
Apple & $60 \%$ & $63 \%$ & 72 & $10^{5}$ \\
\hline
\end{tabular}

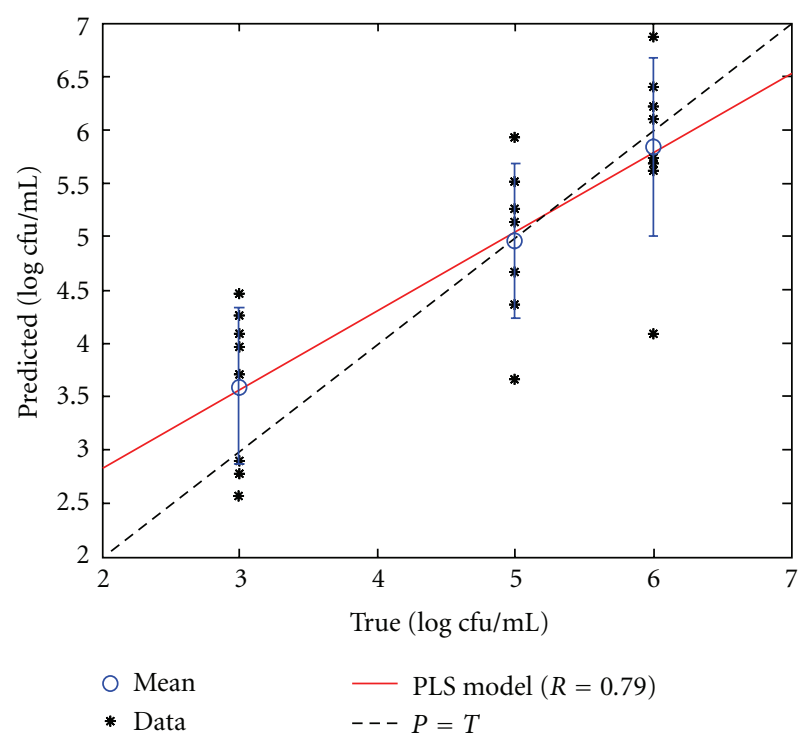

FIGURE 1: PLS regression of EOS data for contaminated orange juice samples owing to predict the amount of contamination (reproduced from [13]).

are sensitive to the change of the global olfactory fingerprint induced by $A$. acidoterrestris presence more than to the guaiacol content of the samples. Yet, this lack of correlation can be regarded as a limitation of the technology since target sensors could certainly facilitate the detection of bacteria in apple juice while enhancing the specificity of the technique.

(b) Early Detection of Microbial Contamination in Processed Tomatoes. Processed tomatoes are a food category extremely exposed to safety risks that can be related to the presence of both chemical residuals, like pesticides and herbicides, and microbial contaminants among which are bacteria and fungi.

To overcome the intrinsic limitations of current quality control protocols that consist in incubating the cans at $30^{\circ} \mathrm{C}$ for 2 weeks and $55^{\circ} \mathrm{C}$ for 1 week in order to favour the microbial growth and gas release which allows for visual inspection of contamination canned-tomatoproducing companies are demanding for tools that allow an early screening of microbial contamination, and, possibly, able to provide an answer in few hours.

The basic idea is that microbial spoilage can alter the headspace through the production of volatiles either rising from the microorganisms' presence or by their metabolism. However, as known, the typical headspace of fresh and canned tomato cannot be ascribed to a few compounds [50, 51], but it depends on a large number of volatiles, the nature, and relative amount of which can be related to the raw matter composition as well as to fermentation or ripening conditions, thus determining the volatile fingerprint of the product. Thus EN was found to be a promising approach to this problem, as illustrated in the following case study.

Concina et al. [35] have investigated the ability of EOS to perform early diagnosis of microbial contamination of canned peeled tomatoes aiming to design an analytical protocol for an objective quality control at the end of the production chain.

A main challenge for the EOS was the classification of contaminated samples subjected to multiple microbial contaminants. Three bacteria (Escherichia coli, Enterobacter cloache, and Lactobacillus plantarum), one yeast (Saccharomyces cerevisiae) and two fungi (Aspergillus carbonarius and Penicillium puberulum) were used in this study to contaminate the product.

Contaminated samples by the different organisms were clustered on the PC2 and PC3 plane (Figure 2) while PC1 was eliminated because it was affected by sensor drift (a wellknow problem that will be discussed in the next section).

Uncontaminated tomato samples (black asterisks in figure) can be separated from the contaminated ones on the PC3. Supervised classification tests were also performed by implementing a 5-fold cross-validated k-NN classifier: the results, obtained by excluding the $\mathrm{PC} 1$ from the classifier inputs, provided the $83 \%$ of correct classification rate for contaminated samples.

This good classification of contaminated samples was possible irrespectively of the type of organism, although different organisms showed peculiar behavior. In particular, E. coli (open squares) and S. cerevisiae (black circles) were much better separated from uncontaminated tomato samples, and they were recognized just after 48 hours of incubation. In fact, the growth time of the microorganisms, reflecting their metabolic kinetics, demonstrated to play a significant role in the headspace composition and hence is reflected on the EOS detection limit.

E. coli is a relevant case since it may become pathogenic at high concentrations or if mutant strains are present. A disappointing behavior was observed with the EOS, that is, the organism resulted is better detectable after 48 hours of growth than after one week, while $S$. cerevisiae continued its growth (see the arrows pictured in Figure 2). It was supposed that this result was related with the preference of the growth medium showed by the organisms which may affect their intrinsic growth rate. Fungi, like S. cerevisiae, have slower growth rate, but they prefer an acidic environment (tomato sauce has a $4.5 \mathrm{pH}$ ). On the other hand, E. coli has a faster metabolism, making it detectable just after 48 hours, but the acid environment is less favorable for the bacteria growth thus reaching probably senescence or quiescence after few days with a consequent decrease of volatile compounds release. 


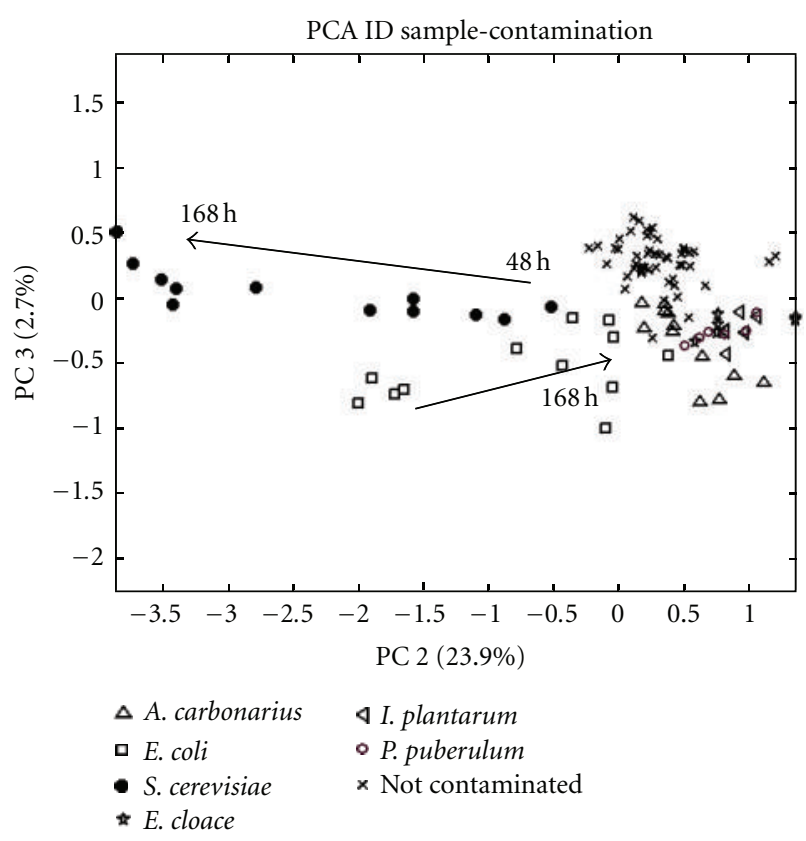

FIGURe 2: PCA plot of EOS data projected on the PC2-PC3 plane showing the discrimination between uncontaminated and contaminated samples (modified from Concina et al. [35]).

(c) Screening Fungal and Fumonisin Contamination of Maize Grains. Maize (Zea mays L.) is a cereal crop spread through the world representing one of the most important food commodities. Unfortunately, it can be easily spoiled by filamentous fungi, particularly those belonging to the genera Aspergillus, Eurotium, Penicillium, and Fusarium, with consequent loss and deterioration of the grains and decrease of their nutritional value.

Importantly the production of toxic fungal secondary metabolites, mycotoxins, which accumulate in cereal grains used as animal feeds and/or human food, can pose a hazard to animal and human health. Indeed, mycotoxins show a toxicity range from acute death to chronic diseases, with different kinds of cancer and immunosuppression being the most important toxic effects. The early detection of the fungal species producing mycotoxins or of the mycotoxins themselves has become very important to prevent the human and animal risk deriving from the entry of mycotoxins into the food chain.

Fusarium spp. are among the most important groups of mycotoxigenic moulds often encountered as contaminants of cereal grains. Specifically, Fusarium verticillioides, the most prevalent pathogen, and $F$. proliferatum are producers of fumonisins, an important group of mycotoxins among whom the most prevalent, which is also believed to be the most toxic, is fumonisins B-1 (FB-1).

Odour mapping techniques are commonly used for quality grading of grains. These controls are routinely performed by a human olfactory panel constituted by inspectors smelling the grain odour. Changes in the emitted volatiles compounds (VOCs) due to mycotoxin contamination [52,
53] or to microbial spoilage of food or feed have already been reported in the literature [54].

In Falasconi et al. [15], the EOS was tested to discriminate fumonisins contamination in maize using four strains of Fusarium verticillioides, among which two strains classified as fumonisin producer. Clear evidence that EN technology can be able to detect maize contamination and to discriminate between fumonisin $\mathrm{B} 1$ producer $(\mathrm{FB} 1+)$ and not producer (FB1-) strains was reported, confirming previous preliminary findings achieved by Keshri and Magan [55].

Importantly, the EOS patterns of the two strains were noted to be identical until the sixth day of growth, but at the seventh day a differentiation was possible by taking the PC2 values of the measured sensor signals. The separation of the two individuals monotonically increased with the vial incubation time, and after 15 hours they were clearly distinguished (Figure 3).

Further validation on a microbiologically larger and statistically significant number of fungal species and strains was carried out by Gobbi et al. [20]. In this study, a comprehensive investigation of 18 fungal strains of 6 Fusarium species has been undergone in order to definitively prove whether the EOS could be effectively applied to detect fungal contaminated maize. In addition the EOS ability to quantitatively discriminate between different fumonisins contents in maize was tested for the first time.

The EOS was able to cluster the maize samples primarily according to the level of fumonisins contamination and independently from the agent of contamination. In fact the strains of $F$. verticillioides and $F$. proliferatum were sorted depending on their actual fumonisins production and not on taxonomic basis, as the low fumonisins producing strains of $F$. verticillioides and proliferatum were grouped with the strains of the not fumonisins producing species.

The obtained results demonstrated that there is a significant correlation between the EOS signals and the fumonisin content of the samples while, on the contrary, no correlation was found between the EOS data and the CFU counts of the samples or either the CFU counts and the fumonisin content. Moreover, the EOS prediction capability of the fumonisins content was ascertained by implementing a cross-validated PLS model (Figure 4) with CD-ELISA test values used as covariates. Although the EOS did not provide a precise fumonisins quantification, high $(>1000 \mathrm{ppm})$ and low $(<2 \mathrm{ppm})$ contamination levels were perfectly recognized; thus in large grain storage facilities the EN method can be effectively used to rapidly identify maize samples having fumonisin content below or well above the legal limit set by the European regulations.

(d) Fungal Contamination of Green Coffee Beans. Fungal growth can occur on green coffee beans along all the distribution chain, eventually bringing on health hazards to consumers, because of production of toxic metabolites like mycotoxins [56]. Besides, the sensorial contamination due to volatiles by-products of fungal metabolism could cause defects on coffee also after roasting. Therefore, it is necessary to develop strategies to distinguish and quantify 


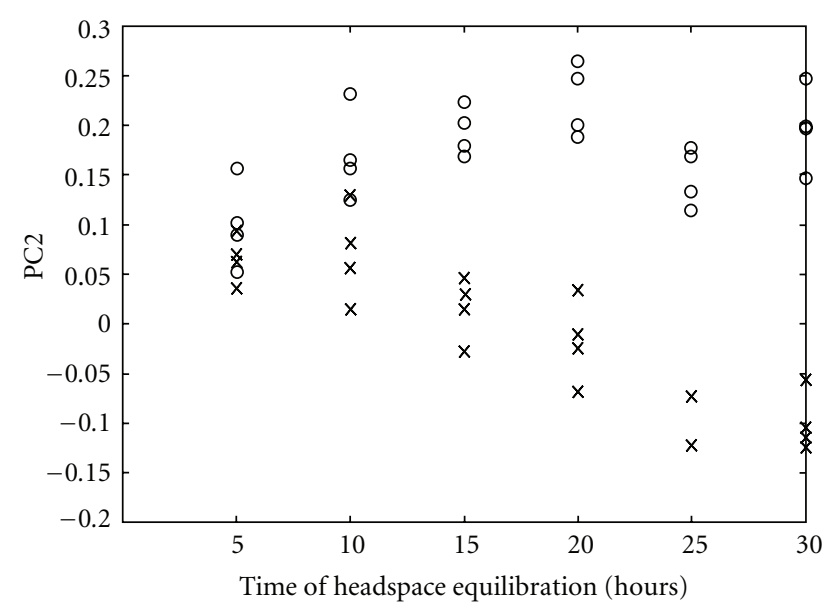

O FB1-

$\times \mathrm{FB} 1+$

Figure 3: Second principal component (PC2) values of the EOS sensor signals plotted against the headspace equilibration time (hours) at seven days of growth of the two F. verticillioides strains (modified from Falasconi et al. [15]).

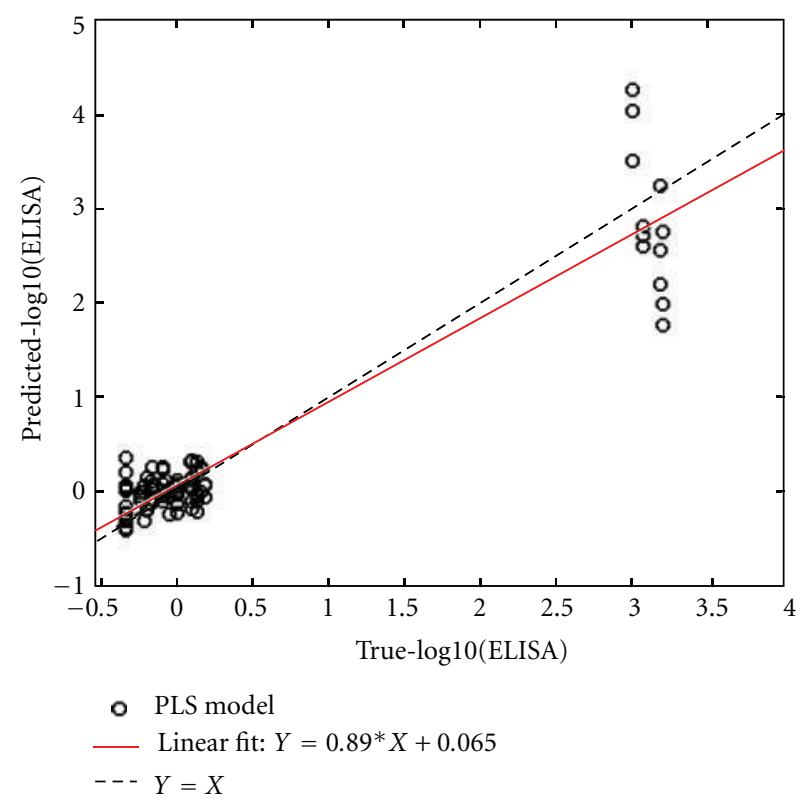

Figure 4: Prediction of fumonisins content in maize cultures by EOS (reproduced from Gobbi et al. [20]).

at early stages fungal infection and toxin production. One of the most promising techniques is the analysis of volatile compounds in the headspace gas surrounding the samples. For this reason, the ability of the EOS to early detect the microbial contamination of Arabica green coffee was evaluated. Original, but preliminary, results are presented in what follows.

The main toxigenic fungal genera (Aspergillus and Penicillium) are natural coffee contaminants and are present from the field to the warehouse [57]. Two species of the genus
Aspergillus (A.niger type strain A733 and A. ochraceus type strain DSM 824) were selected. The green coffee beans were first contaminated and then incubated in a moist chamber at $27^{\circ} \mathrm{C}$ for 11 days (analysed at $0,4,6,8$, and 11 days after inoculations) in order to promote the growth of fungi inoculated and to standardized at 0,6 the activity water (Aw). Coffee beans were preliminarily sterilized with UV light in order to remove any undesired contamination and to be sure of having sterile control samples.

The two selected strains were tested in parallel with classical microbiological isolation techniques to quantify the actual contamination in the days after inoculation and with analytical chemical techniques, like gas chromatography coupled with mass spectroscopy (GC-MS) with solid-phase microextraction (SPME), for the detection of formation of any secondary metabolites.

GCMS analyses evidenced quite different chemical volatile profiles for uncontaminated and contaminated coffee beans (Figure 5). In particular, a relevant presence of carbon dioxide, ethanol, and ethyl acetate, which are considered to be typical microbial metabolites, emerged in the headspace of contaminated samples. Samples contamination was also confirmed by the appearance of anisole, recognized as one of the major volatile contaminants in fungal contaminated coffee [58]. The EOS was able to successfully discriminate contaminated samples of green coffee from noncontaminated ones (Figure 6). Five/six days of growth were necessary to identify the coffee contamination; this was attained by means of the monitoring of mold growth on Petri dishes.

\section{Discussion and Issues}

Electronic nose could be very valuable tools to evaluate microbiological food quality and safety. Strengths of the electronic nose include good sensitivity and correlation with data from microbiological tests. It might have other advantages regarding portability, price, and ease of use. Therefore, it has the potential to move from well-equipped chemical laboratories to industrial routine at-line controls. At present ENs present some downsides that must be overcome, however.

The major limitation of currently available chemical sensor-based ENs remains the independence (crosscorrelation) and selectivity of the sensing devices. Sensors with poor selectivity affect adversely the discriminating power of the array. Moreover, the EN use of semiselective sensors prevents any real identification or quantification of the individual compounds present in the headspace of a food sample, as it can be done with classical analytical techniques, which sometimes can be crucial for the enduser. In fact, whenever specific contamination markers are present (as shown in our first case study), their detection allows more reliable, faster, and more replicable results than training an EN. To tackle this issue, in recent years, classical chemosensor technologies were complemented by new emerging technologies [43]. In particular, machine olfaction has benefited from developments in several fields 


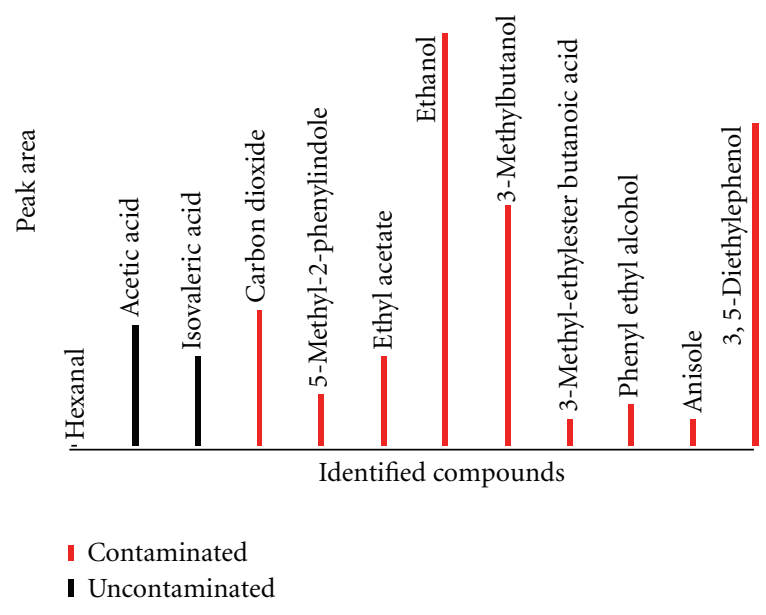

Figure 5: Comparison between the GC-MS spectra (most abundant compounds) of contaminated and uncontaminated samples.

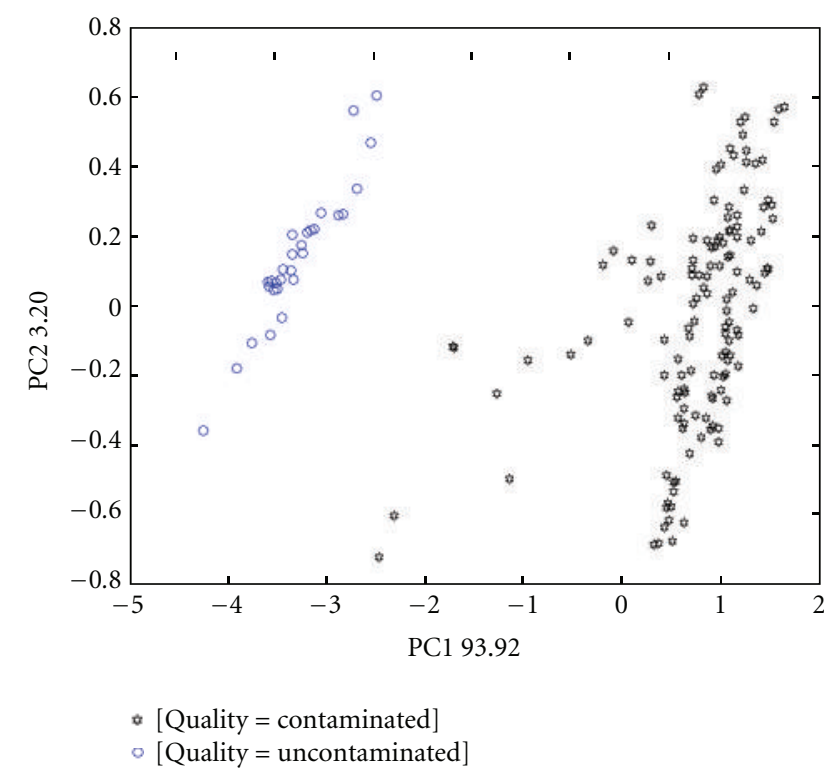

FIGURE 6: PCA score plot of contaminated green coffees beans data.

ranging from optical technologies developed by the telecommunications industry to the improvements in analytical chemistry such as gas chromatography, mass spectroscopy, and ion mobility spectrometry. This trend has also narrowed the gap between traditional ENs-used as a black boxand classical analytical techniques which aim to quantify individual volatile components. Nonetheless, it is unrealistic to envisage a universal electronic nose that is able to cope with every odour type; conversely data processing and instrumentation must be specifically designed for each application.

The EN training procedure still remains lengthy and laborious, and, finally, the lack of sensors stability and reproducibility over time can put at risk the use of previously collected databases, which are compulsory for data comparison purposes and for the classification of new unknown samples. The problem of chemical sensor stability over time is known as "sensor drift." It consists of (more or less) small and nondeterministic temporal variations of the sensor response when it is exposed to the same analytes under identical conditions. This is generally attributed to sensors aging or thermomechanical degradation, but it can also be influenced by a variety of sources including environmental factors. The main result is that sensors selectivity and sensitivity slowly decrease with time. Drift correction is perhaps one the most relevant issues in the field of chemical sensors. Indeed, in spite of constant improvements in micro/nanofabrication techniques that allowed the production of sensing devices with superior stability, it is still impossible to fabricate chemical sensors without drift. This issue is currently approached on one hand by improving the sensors performances with novel sensing materials [59], on the other hand by adopting various approaches for compensating sensors drift to increase pattern recognition accuracy $[60,61]$.

Another major drawback, partially connected to the previous issue, is that in case of sensor breakage it would be very difficult to replace the sensor by another one having exactly the same behaviour. This dramatically jeopardizes the use of previously collected databases, which are compulsory for data comparison purposes and for the classification of new unknown samples. This problem has been addressed from different points of view: while researchers are trying to understand the mechanisms related to materials and device processing to improve the sensors reproducibility, at industrial level this problem is fixed by producing a large number of sensors and then by selecting the most similar on the basis of an application specific test protocol. The problem of database recovering after sensors replacement in an electronic nose has been also approached by means of multivariate calibration methods [62]. Such methods permit to alleviate the problems due to lack of sensor-to-sensor reproducibility and allow recovering good classification rates.

\section{Conclusions}

In this work, some relevant applications of electronic nose technology to microbiological food quality control have been reviewed. The literature review has been accompanied by some significant case studies previously performed in this field by the same authors, in order to provide the reader a better insight of the EN application. All the reported case studies showed promising results, thus confirming that EN could represent a rapid mean for controlling and improving the microbiological quality of food. Further investigations to induce more sensitive specific response of the gas sensors may help both in the qualitative and quantitative analysis of microbial contamination, however.

Before the EN can be treated as a completely reliable, industrial instrument, to be used in the food field, much improvement is still needed on the technology side, for instance, improve sensor selectivity, reduce interferences (e.g., to humidity), compensate drift effects, and handle with sensor replacement. 
Progresses could also be made on the application side by better investigation of available technologies. At present, the major part of EN applications is represented by limited feasibility studies, often present as preliminary results, with poor validation especially in terms of reproducibility and predictive ability. Few replication or confirmation studieslike the two cases (a) and (c) presented here-are reported in the literature; conversely for people working in the field would be very beneficial to see long term studies results with extensive investigation of data reproducibility and system stability.

Keeping in mind advantages and limitations, ENs do not allow to replace human panels or analytical techniques, as long as their ability to smelling odors rather than detecting and quantifying specific volatiles is still far from required standards. However, they can be used in parallel to those techniques, or even considered as valuable alternatives, to perform quick "go-no go" product tests or occasionally replace human panels when nonodorous, irritant, or potentially toxic volatile substances need to be detected.

\section{Acknowledgments}

The authors wants to acknowledge for support the FIRB project "Rete Nazionale di Ricerca sulle Nanoscienze ItalNanoNet," Protocollo: RBPR05JH2P, 2009-2013, Ministero dell'Istruzione, dell'Università e della Ricerca.

\section{References}

[1] M. J. Saxby, Food Taints and Off-flavours, Blackie Academic \& Professional, Cambridge, UK, 1st edition, 1993.

[2] L. M. Reid, C. P. O’Donnell, and G. Downey, "Recent technological advances for the determination of food authenticity," Trends in Food Science and Technology, vol. 17, no. 7, pp. 344353, 2006.

[3] K. Arora, S. Chand, and B. D. Malhotra, "Recent developments in bio-molecular electronics techniques for food pathogens," Analytica Chimica Acta, vol. 568, no. 1-2, pp. 259-274, 2006.

[4] M. Pardo and G. Sberveglieri, "Electronic olfactory systems based on metal oxide semiconductor sensor arrays," MRS Bulletin, vol. 29, no. 10, pp. 703-701, 2004.

[5] T. C. Pearce, S. S. Shiffman, H. T. Nagle, and J. W. Gardner, Handbook of Machine Olfaction, Wiley-VHC, Weinheim, Germany, 2003.

[6] E. Schaller, J. O. Bosset, and F. Escher, "“Electronic noses" and their application to food," Food Science and Technology, vol. 31, no. 4, pp. 305-316, 1998.

[7] M. Ghasemi-Varnamkhasti, S. S. Mohtasebi, and M. Siadat, "Biomimetic-based odor and taste sensing systems to food quality and safety characterization: an overview on basic principles and recent achievements," Journal of Food Engineering, vol. 100, no. 3, pp. 377-387, 2010.

[8] A. Berna, "Metal oxide sensors for electronic noses and their application to food analysis," Sensors, vol. 10, no. 4, pp. 38823910, 2010.

[9] A. D. Wilson and M. Baietto, "Applications and advances in electronic-nose technologies," Sensors, vol. 9, no. 7, pp. 5099$5148,2009$.
[10] M. Peris and L. Escuder-Gilabert, "A 21st century technique for food control: electronic noses," Analytica Chimica Acta, vol. 638, no. 1, pp. 1-15, 2009.

[11] N. Sahgal, R. Needham, F. J. Cabañes, and N. Magan, "Potential for detection and discrimination between mycotoxigenic and non-toxigenic spoilage moulds using volatile production patterns: a review," Food Additives and Contaminants, vol. 24, no. 10, pp. 1161-1168, 2007.

[12] N. Magan and P. Evans, "Volatiles as an indicator of fungal activity and differentiation between species, and the potential use of electronic nose technology for early detection of grain spoilage," Journal of Stored Products Research, vol. 36, no. 4, pp. 319-340, 2000.

[13] S. Cagnasso, M. Falasconi, M. P. Previdi et al., "Rapid screening of alicyclobacillus acidoterrestris spoilage of fruit juices by electronic nose: a confirmation study," Journal of Sensors, vol. 2010, Article ID 143173, 2010.

[14] S. Labreche, S. Bazzo, S. Cade, and E. Chanie, "Shelf life determination by electronic nose: application to milk," Sensors and Actuators B, vol. 106, no. 1, pp. 199-206, 2005.

[15] M. Falasconi, E. Gobbi, M. Pardo, M. Della Torre, A. Bresciani, and G. Sberveglieri, "Detection of toxigenic strains of Fusarium verticillioides in corn by electronic olfactory system," Sensors and Actuators B, vol. 108, no. 1-2, pp. 250257, 2005.

[16] J. Olsson, T. Börjesson, T. Lundstedt, and J. Schnürer, "Volatiles for mycological quality grading of barley grains: determinations using gas chromatography-mass spectrometry and electronic nose," International Journal of Food Microbiology, vol. 59, no. 3, pp. 167-178, 2000.

[17] R. Paolesse, A. Alimelli, E. Martinelli et al., "Detection of fungal contamination of cereal grain samples by an electronic nose," Sensors and Actuators B, vol. 119, no. 2, pp. 425-430, 2006.

[18] P. Evans, K. C. Persaud, A. S. McNeish, R. W. Sneath, N. Hobson, and N. Magan, "Evaluation of a radial basis function neural network for the determination of wheat quality from electronic nose data," Sensors and Actuators B, vol. 69, no. 3, pp. 348-358, 2000.

[19] F. Cheli, A. Campagnoli, L. Pinotti, G. Savoini, and V. Dell'Orto, "Electronic nose for determination of aflatoxins in maize," Biotechnology, Agronomy and Society and Environment, vol. 13, pp. 39-43, 2009.

[20] E. Gobbi, M. Falasconi, E. Torelli, and G. Sberveglieri, "Electronic nose predicts high and low fumonisin contamination in maize cultures," Food Research International, vol. 44, pp. 992999, 2011.

[21] M. Falasconi, Development and validation of an Electronic Olfactory System for the food industry, Ph.D. thesis, University of Brescia, Italy, 2004.

[22] S. Marín, M. Vinaixa, J. Brezmes et al., "Use of a MSelectronic nose for prediction of early fungal spoilage of bakery products," International Journal of Food Microbiology, vol. 114, no. 1, pp. 10-16, 2007.

[23] R. Needham, J. Williams, N. Beales, P. Voysey, and N. Magan, "Early detection and differentiation of spoilage of bakery products," Sensors and Actuators B, vol. 106, no. 1, pp. 20-23, 2005.

[24] S. Panigrahi, S. Balasubramanian, H. Gu, C. Logue, and M. Marchello, "Neural-network-integrated electronic nose system for identification of spoiled beef," Food Science and Technology, vol. 39, no. 2, pp. 135-145, 2006. 
[25] S. Balasubramanian, S. Panigrahi, C. M. Logue, C. Doetkott, M. Marchello, and J. S. Sherwood, "Independent component analysis-processed electronic nose data for predicting Salmonella typhimurium populations in contaminated beef," Food Control, vol. 19, no. 3, pp. 236-246, 2008.

[26] N. El Barbri, E. Llobet, N. El Bari, X. Correig, and B. Bouchikhi, "Electronic nose based on metal oxide semiconductor sensors as an alternative technique for the spoilage classification of red meat," Sensors, vol. 8, no. 1, pp. 142-156, 2008.

[27] D. Wang, X. Wang, T. Liu, and Y. Liu, "Prediction of total viable counts on chilled pork using an electronic nose combined with support vector machine," Meat Science, vol. 90, no. 2, pp. 373-377, 2012.

[28] G. Olafsdottir, E. Chanie, F. Westad et al., "Prediction of microbial and sensory quality of cold smoked Atlantic salmon (Solmo salar) by electronic nose," Journal of Food Science, vol. 70, no. 9, pp. S563-S574, 2005.

[29] W. X. Du, C. M. Lin, T. Huang, J. Kim, M. Marshall, and C. I. Wei, "Potential application of the electronic nose for quality assessment of salmon fillets under various storage conditions," Journal of Food Science, vol. 67, no. 1, pp. 307-313, 2002.

[30] J. Chantarachoti, A. C. M. Oliveira, B. H. Himelbloom, C. A. Crapo, and D. G. McLachlan, "Portable electronic nose for detection of spoiling alaska pink salmon (Oncorhynchus gorbuscha)," Journal of Food Science, vol. 71, no. 5, pp. S414S421, 2006.

[31] N. E. Barbri, J. Mirhisse, R. Ionescu et al., "An electronic nose system based on a micro-machined gas sensor array to assess the freshness of sardines," Sensors and Actuators B, vol. 141, no. 2, pp. 538-543, 2009.

[32] S. Benedetti, S. Iametti, F. Bonomi, and S. Mannino, "Head space sensor array for the detection of aflatoxin M1 in raw ewe's milk," Journal of Food Protection, vol. 68, no. 5, pp. 10891092, 2005.

[33] C. Li, N. E. Schmidt, and R. Gitaitis, "Detection of onion postharvest diseases by analyses of headspace volatiles using a gas sensor array and GC-MS," Food Science and Technology, vol. 44, no. 4, pp. 1019-1025, 2011.

[34] I. Concina, M. Falasconi, E. Gobbi et al., "Early detection of microbial contamination in processed tomatoes by electronic nose," Food Control, vol. 20, no. 10, pp. 873-880, 2009.

[35] E. Gobbi, M. Falasconi, I. Concina et al., "Electronic nose and Alicyclobacillus spp. spoilage of fruit juices: an emerging diagnostic tool," Food Control, vol. 21, no. 10, pp. 1374-1382, 2010.

[36] I. Concina, M. Bornšek, S. Baccelliere, M. Falasconi, E. Gobbi, and G. Sberveglieri, "Alicyclobacillus spp.: detection in soft drinks by Electronic Nose," Food Research International, vol. 43, no. 8, pp. 2108-2114, 2010.

[37] A. Z. Berna, S. Trowell, W. Cynkar, and D. Cozzolino, "Comparison of metal oxide-based electronic nose and mass spectrometry-based electronic nose for the prediction of red wine spoilage," Journal of Agricultural and Food Chemistry, vol. 56, no. 9, pp. 3238-3244, 2008.

[38] S. Balasubramanian, S. Panigrahi, B. Kottapalli, and C. E. Wolf-Hall, "Evaluation of an artificial olfactory system for grain quality discrimination," Food Science and Technology, vol. 40, no. 10, pp. 1815-1825, 2007.

[39] J. Olsson, T. Börjesson, T. Lundstedt, and J. Schnürer, "Detection and quantification of ochratoxin A and deoxynivalenol in barley grains by GC-MS and electronic nose," International Journal of Food Microbiology, vol. 72, no. 3, pp. 203-214, 2002.
[40] A. Jonsson, F. Winquist, J. Schnurer, H. Sundgren, and I. Lundstrom, "Electronic nose for microbial quality classification of grains," International Journal of Food Microbiology, vol. 35, no. 2, pp. 187-193, 1997.

[41] J. E. Haugen, K. Rudi, S. Langsrud, and S. Bredholt, "Application of gas-sensor array technology for detection and monitoring of growth of spoilage bacteria in milk: a model study," Analytica Chimica Acta, vol. 565, no. 1, pp. 10-16, 2006.

[42] N. Magan, A. Pavlou, and I. Chrysanthakis, "Milk-sense:a volatile sensing system recognizes spoilage bacteria and yeasts in milk," Sensors and Actuators B, vol. 72, no. 1, pp. 28-34, 2001.

[43] F. Röck, N. Barsan, and U. Weimar, "Electronic nose: current status and future trends," Chemical Reviews, vol. 108, no. 2, pp. 705-725, 2008.

[44] D. L. Massart, B. G. M. Vandeginste, L. M. C. Buydens, S. De Jong, P. J. Lewi, and J. Smeyers-Verbeke, "Principal components," in Handbook of Chemometrics and Qualimetrics, Part A, chapter 17, Elsevier, Amsterdam, The Netherlands, 1997.

[45] M. Pardo and G. Sberveglieri, "Classification of electronic nose data with support vector machines," Sensors and Actuators B, vol. 107, no. 2, pp. 730-737, 2005.

[46] D. L. Massart, B. G. M. Vandeginste, L. M. C. Buydens, S. De Jong, P. J. Lewi, and J. Smeyers-Verbeke, "Quantitative structure activity relationships (QSARs)," in Handbook of Chemometrics and Qualimetrics, Part B, chapter 37, Elsevier, Amsterdam, The Netherlands, 1997.

[47] M. Walker and C. A. Phillips, "Alicyclobacillus acidoterrestris: an increasing threat to the fruit juice industry?" International Journal of Food Science and Technology, vol. 43, no. 2, pp. 250260, 2008.

[48] S. S. Chang and D. H. Kang, "Alicyclobacillus spp. in the fruit juice industry: history, characteristics, and current isolation/detection procedures," Critical Reviews in Microbiology, vol. 30, no. 2, pp. 55-74, 2004.

[49] N. Jensen and F. B. Whitfield, "Role of Alicyclobacillus acidoterrestris in the development of a disinfectant taint in shelf-stable fruit juice," Letters in Applied Microbiology, vol. 36, no. 1, pp. 9-14, 2003.

[50] A. Z. Berna, J. Lammertryn, S. Saevels, C. Di Natale, and B. M. Nicolaï, "Electronic nose systems to study shelf life and cultivar effect on tomato aroma profile," Sensors and Actuators B, vol. 97, no. 2-3, pp. 324-333, 2004.

[51] F. Maul, S. A. Sargent, M. O. Balaban, E. A. Baldwin, D. J. Huber, and C. A. Sims, "Aroma volatile profiles from ripe tomatoes are influenced by physiological maturity at harvest: an application for electronic nose technology," Journal of the American Society for Horticultural Science, vol. 123, no. 6, pp. 1094-1101, 1998.

[52] H. J. Zeringue, D. Bhatnagar, and T. E. Cleveland, "C15H24 volatile compounds unique to aflatoxigenic strains of Aspergillus flavus," Applied and Environmental Microbiology, vol. 59, no. 7, pp. 2264-2270, 1993.

[53] H. H. Jelen, C. J. Mirocha, E. Wasowicz, and E. Kaminski, "Production of volatile sesquiterpenes by Fusarium sambucinum strains with different abilities to synthesize trichothecenes," Applied and Environmental Microbiology, vol. 61, no. 11, pp. 3815-3820, 1995.

[54] D. Hodgins and D. Simmonds, "Sensory technology for flavor analysis," Cereal Foods World, vol. 40, pp. 186-191, 1995.

[55] G. Keshri and N. Magan, "Detection and differentiation between mycotoxigenic and non-mycotoxigenic strains of two Fusarium spp. using volatile mycotoxigenic strains of 
two production profiles and hydrolytic enzymes," Journal of Applied Microbiology, vol. 89, no. 5, pp. 825-833, 2000.

[56] R. A. Etzel, "Mycotoxins," Journal of the American Medical Association, vol. 287, no. 4, pp. 425-427, 2002.

[57] M. Nakajima, H. Tsubouchi, M. Miyabe, and Y. Ueno, "Survey of aflatoxin B1 and ochratoxin a in commercial green coffee beans by high-performance liquid chromatography linked with immunoaffinity chromatography," Food and Agricultural Immunology, vol. 9, no. 2, pp. 77-83, 1997.

[58] K. Karlshøj, P. V. Nielsen, and T. O. Larsen, "Differentiation of closely related fungi by electronic nose analysis," Journal of Food Science, vol. 72, no. 6, pp. M187-M192, 2007.

[59] E. Comini, G. Faglia, and G. Sberveglieri, Solid State Gas Sensing, Springer, New York, NY, USA, 2008.

[60] M. Padilla, A. Perera, I. Montoliu, A. Chaudry, K. Persaud, and S. Marco, "Drift compensation of gas sensor array data by Orthogonal Signal Correction," Chemometrics and Intelligent Laboratory Systems, vol. 100, no. 1, pp. 28-35, 2010.

[61] S. Di Carlo, M. Falasconi, E. Sanchez, A. Scionti, G. Squillero, and A. Tonda, "Increasing pattern recognition accuracy for chemical sensing by evolutionary based drift compensation," Pattern Recognition Letters, vol. 32, no. 13, pp. 1594-1603, 2011.

[62] O. Tomic, T. Eklöv, K. Kvaal, and J. E. Haugen, "Recalibration of a gas-sensor array system related to sensor replacement," Analytica Chimica Acta, vol. 512, no. 2, pp. 199-206, 2004. 


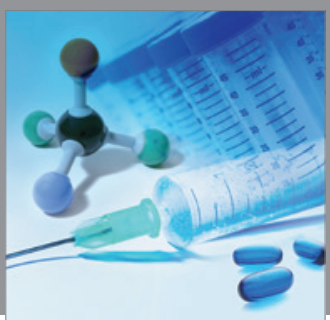

International Journal of

Medicinal Chemistry

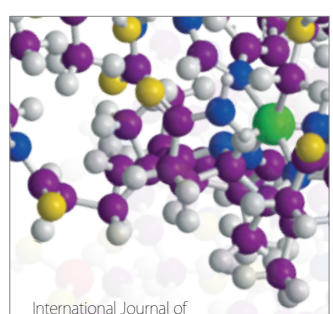

Carbohydrate Chemistry

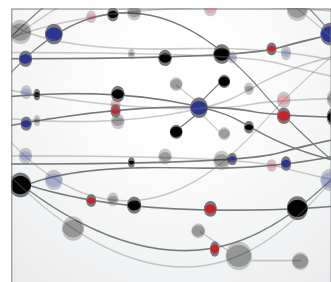

The Scientific World Journal
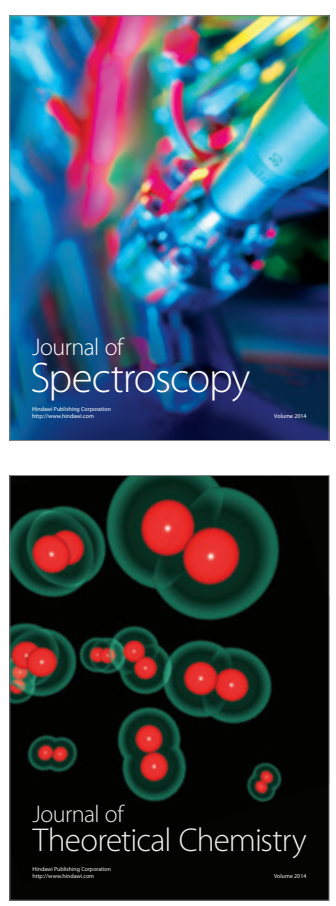
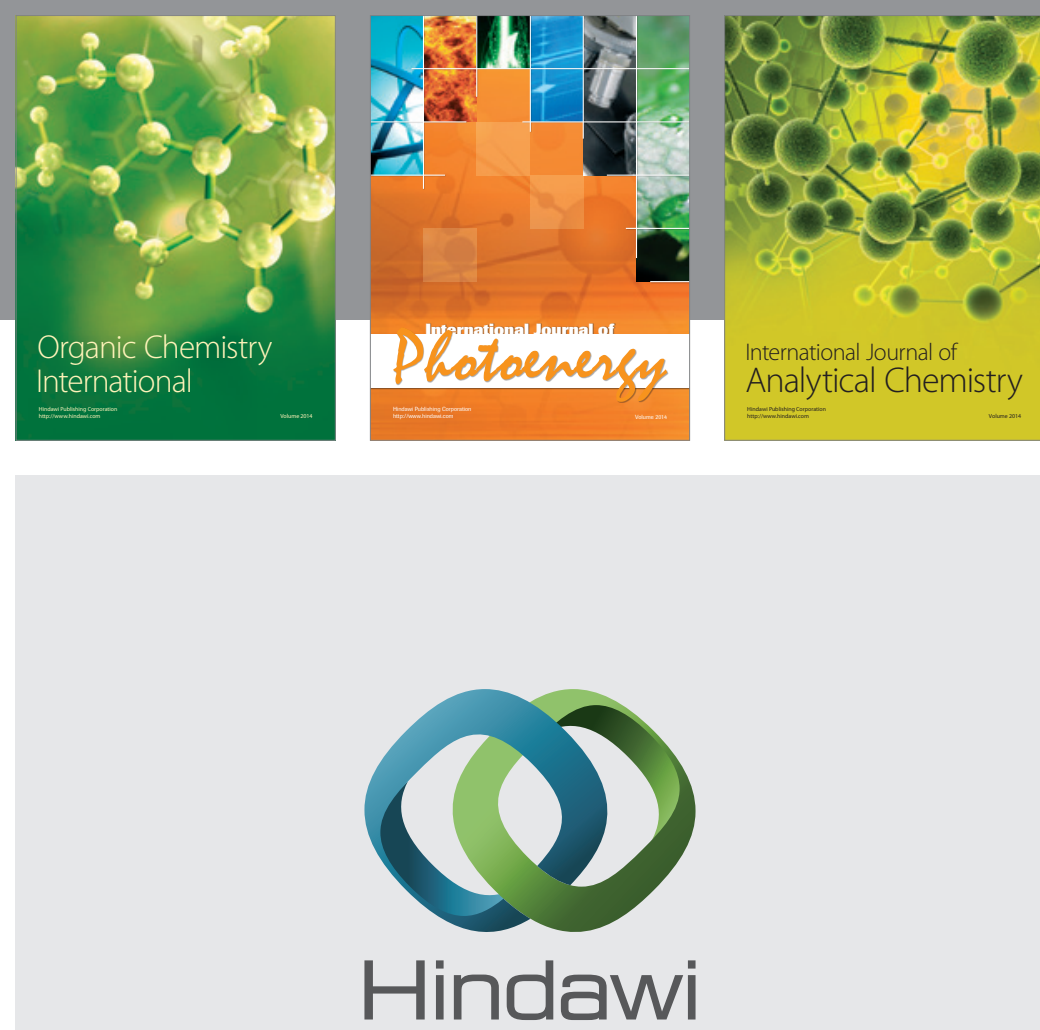

Submit your manuscripts at

http://www.hindawi.com
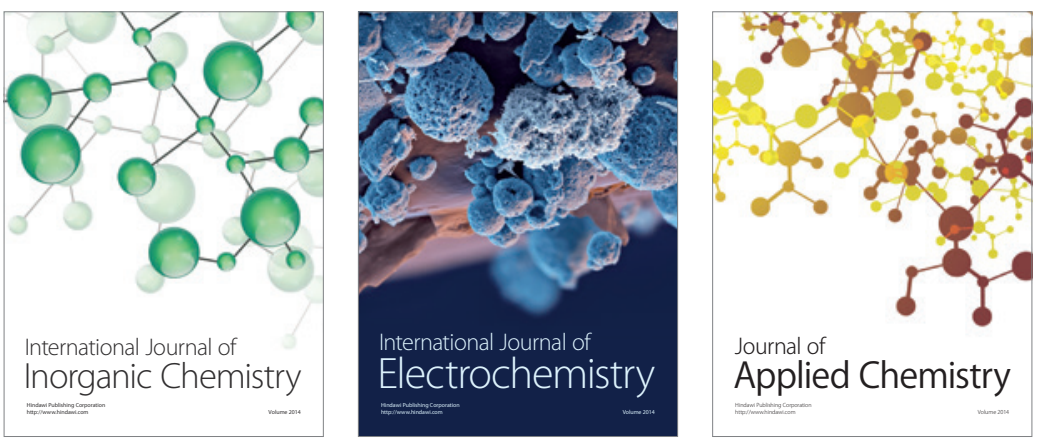

Journal of

Applied Chemistry
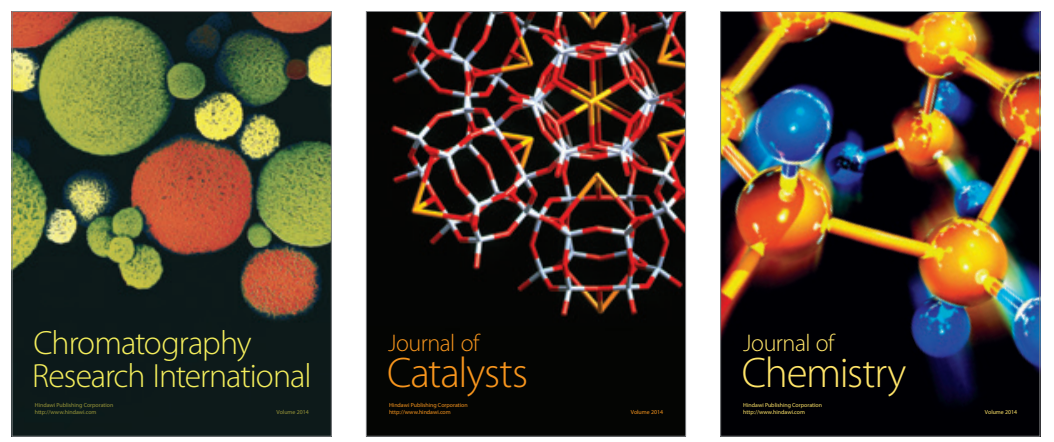
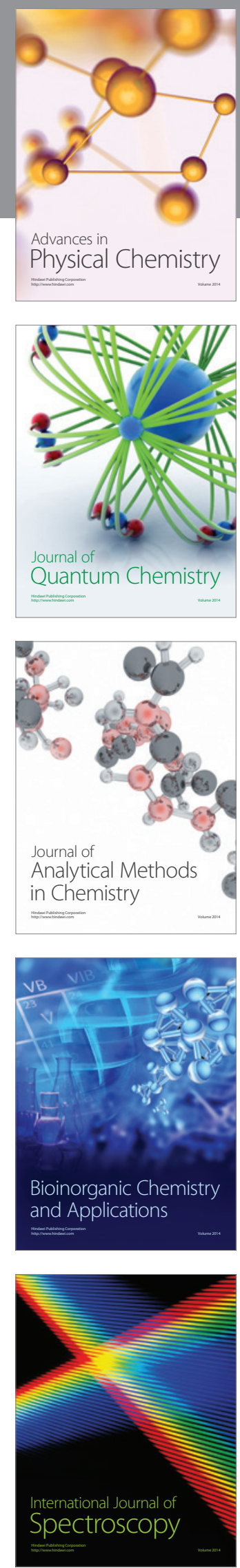